\title{
El uso de analogías y modelos analógicos en La Enseñanza de la Química
}

John Alejandro Torres Díaz

john_torres_pedagogica@yahoo.es

\section{Resumen}

Este trabajo aborda algunas de las características que tendría una propuesta basada en la investigación y desarrollo de analogías que promuevan y faciliten el aprendizaje de las ciencias, en general, y de la química, en particular.

Mediante el desarrollo de la propuesta se pretende generar en los estudiantes una evolución conceptual. Para ello, se presentarán las diferentes temáticas propuestas en el plan de estudios mediante analogías sencillas que puedan ser asimiladas por los estudiantes.

Si bien es cierto que el lenguaje que se utiliza para presentar los diferentes tópicos es complejo y, en algunos casos, abstracto (para los estudiantes, más no para los docentes), se hace necesario utilizar un lenguaje que sea más afín, cotidiano o, en otras palabras, que sea más familiar a los estudiantes. (Galagovsky, L. y Adúriz-Bravo 2001).

\section{Palabras clave}

Analogía, enseñanza de las ciencias, aprendizaje significativo, constructivismo, modelo analógico.

2. Proyecto de Práctica Pedagógica y Didáctica II, primer semestre 2005.

3. Estudiante del Departamento de Química - UPN 


\section{Introducción}

Con frecuencia, explicar la construcción de estructuras conceptuales a partir de otras más simples da lugar a inconvenientes y en algunos casos a discrepancias (Bareiter 1985), sin embargo una de las estrategias cognitivas, la analogía, que en sentido amplio, significa una semejanza formal y parcial entre dos o más representaciones de aspectos de la realidad, ayudan a uno de ellos (el relativo a aspectos mejor conocidos), al otro (el menos conocido o más abstracto), con el objeto de organizar, estructurar y representar un conjunto distinto de aspectos. (Arcá, M. y Guidoni 1989; Galagovsky, L. y Adúriz-Bravo 2001,).

Para (Oliva, J. M. 2001) “las analogías son comparaciones entre dominios de conocimientos que mantienen una cierta relación de semejanza entre sí, constituyen una herramienta frecuente en el pensamiento ordinario de las personas, y ocupan un lugar importante en el ámbito de la enseñanza de las ciencias".

\section{Problema}

Se podría concretar un aprendizaje significativo (Ausubel 1983), o como llamaría Gallego Badillo R, Royman Pérez M. y Torres de Gallego L. N. 1997 Aprendizaje total, del contexto químico, utilizando como matriz la estrategia pedagógica y didáctica fundamentada en el desarrollo y uso de analogías (Galagovsky, L. 2001; Oliva, J.M. y otros 2001; Vasini, E. Y Donati, E. 2001), de corte físico, biológico y ambiental, para la presentación inicial de conceptos, principios y teorías químicas, a una comunidad académica novata, utilizando como medio de comunicación un lenguaje común o "cotidiano" en contraposición al lenguaje científico o "erudito" que generalmente es utilizado en las aulas de clase para la enseñanza de las ciencias.

\section{Preguntas guía}

¿Qué implicaciones tiene el uso de analogías en la enseñanza de las ciencias?, ¿Se podría generar un cambio conceptual, actitudinal, metodológico y axiológico con el uso de analogías? ¿Serían útiles y efectivas las analogías como recurso para la enseñanza de las ciencias?

\section{Antecedentes}

En su trabajo Oliva y otros (2001) muestran que las analogías son comparaciones, símiles o metáforas entre concepciones que mantienen cierto grado de relación y que constituyen una herramienta frecuente en el pensamiento de las personas, así como para la enseñanza en general y en la enseñanza de las ciencias en particular, ya que sirven para comprender hechos o fenómenos que ocurren en nuestro entorno y que resultan más conocidos y familiares a los estudiantes. En términos de Ausubel, las analogías se conciben como los organizadores previos.

En su trabajo Arca, M. Y Guidoni, P. (1989) hacen un análisis del término modelo, el objetivo general del modelo que según su concepción "es un instrumento mental, especialmente apto para la comprensión de la estructura de la realidad", así mismo trata sobre la construcción del conocimiento por medio de modelos, de hecho, "todas las construcciones cognitivas y culturales realizadas por las sociedades humanas son series de modelos del mundo", también hace referencia al término analogía para mostrar relaciones entre dos o más representaciones (modelos) de la realidad.

El artículo muestra como nuestras concepciones ultimas del mundo son producto de la progresión en complejidad de modelos, los primeros modelos se presentan en edades tempranas, éstos generalmente son errados, aunque parezcan lógicas para ellos, en un segundo estadio se generan modelos infantiles (que son aún más complejos que los primeros modelos). Además, es casi imposible inventar un modelo 
abstracto, ficticio o artificial de una temática compleja en edades tempranas.

Las diferentes investigaciones relacionadas con el uso de analogías han concluido que algunas de las dificultades y problemas son:

* Las analogías pueden no ser suficientemente familiares.

* Las analogías pueden ser más complejas que la realidad estudiada.

* Las analogías pueden generar concepciones erróneas, que se pueden afianzar aún más si son visualizadas por varios estudiantes.

* La interpretación literal de una analogía puede conducir a un razonamiento rígido que puede provocar dificultades en un aprendizaje posterior.

* A veces el estudiante se queda con el hecho anecdótico antes que el principio o la razón de ser que subyace en la analogía.

* Muchas de las analogías propuestas no se pueden llevar a la práctica porque no son fácilmente representables.

\section{Referente teórico}

Desde un punto de vista educativo, sirven para comprender una determinada noción o fenómeno, que se denomina problema o blanco, a través de las relaciones que establece con un sistema análogo al cual se denomina ancla y que resulta para el alumno más conocido y familiar.

Entre las características que deben tener las analogías y que constituyen en marco de referencia para la presente investigación, se pueden mencionar:

* Las analogías deben ser más accesibles que la "realidad"; por ello estas tienen que ser más cotidianas, más familiares a los estudiantes.

* Las representaciones visuales son un factor importante para la comprensión de las analo- gías y, por lo tanto, en la medida en que sea posible su construcción visual, se mejorarán las condiciones para su asimilación.

* Las analogías imaginarias no son muy recomendables para estudiantes que empiezan su formación académica.

* Las analogías propuestas deben simplificarse en lo posible (no es recomendable utilizar una analogía compleja donde intervienen muchos factores y variables para presentar una temática específica).

\section{Objetivo general}

Desarrollar una metodología de enseñanzaaprendizaje, en la que la presentación de conceptos, principios y teorías No se lleve a cabo de la manera tradicional, sino por medio del desarrollo, uso y análisis de analogías.

\section{Metodología}

Se trabajó con un grupo de estudiantes que están cursando grado $10^{\circ}$, sección 02 , que está constituido por 41 estudiantes (hombres y mujeres) cuyas edades oscilan entre 14 - 17 años.

El proyecto se pondrá en práctica en la IED "Cultura Popular", ubicado en el sector de ciudad Montes, Bogotá.

Las pruebas escritas son instrumentos que sirven para la recolección de datos (información), en nuestro contexto, éstas serán de carácter cualitativo.

Para escudriñar las pre-concepciones de los estudiantes y a su vez conocer como establecen relaciones entre los conceptos presentados en el aula de clases, se desarrollaran una serie de talleres que estarán acordes con la temática de clase, éstas estarán circunscritas bajo ciertos enunciados que tendrán un componente adicional: Las preguntas no se plantearán de la manera tradicional, sino que estarán enmarcadas 
en un contexto cotidiano, los cuales crearán un puente entre la información dada y un hecho o situación que ocurre en nuestro entorno.

Para conocer las concepciones que tienen los estudiantes del grado 10 - 02 del I.E.D. "Cultura Popular" en torno a lo que ellos conciben como analogías o modelos analógicos, y su importancia en la enseñanza de las ciencias en general y de la química en particular, se ha diseñado y aplicado un cuestionario constituido por trece (13) interrogantes los cuales se dividen en tres categorías:

1. Interpretación de relaciones análogas a situaciones cotidianas.

2. Concepción acerca de lo que es una analogía.
3. Prospectiva del uso de analogías en la enseñanza de la Química.

Las preguntas 1-7, indagan acerca de cómo los estudiantes (hombres y mujeres) evidencian la generación de relaciones análogas a situaciones cotidianas con modelos gráficos o análogos que tienen una similitud con una situación real.

Las preguntas 8, 9 y 10 tienen como finalidad encontrar las concepciones acerca de lo que son las analogías, en que situaciones se podrían poner en practica y si se facilita su asimilación.

Los últimos tres cuestionamientos 11, 12 y 13, pretenden visualizar si el uso de analogías es pertinente en la enseñanza de la química.

\section{Resultados - Análisis}

Tabla 1. Interpretación de relaciones análogas a situaciones cotidianas.

\begin{tabular}{|c|c|c|c|c|c|c|c|c|}
\hline \multirow[t]{2}{*}{ Pregunta } & \multicolumn{2}{|c|}{$\begin{array}{c}\text { Buena interpretación } \\
\text { del modelo } \\
\text { análogo \% }\end{array}$} & \multicolumn{2}{|c|}{$\begin{array}{c}\text { Incorrecta } \\
\text { interpretación del } \\
\text { modelo análogo \% }\end{array}$} & \multicolumn{2}{|c|}{$\begin{array}{l}\text { Mala interpretación del } \\
\text { modelo análogo \% }\end{array}$} & \multicolumn{2}{|c|}{$\begin{array}{l}\text { No hay interpretación } \\
\text { del modelo análogo \% }\end{array}$} \\
\hline & Hombre & Mujer & Hombre & Mujer & Hombre & Mujer & Hombre & Mujer \\
\hline 1 & 73 & 100 & 15 & 0 & 0 & 0 & 12 & 0 \\
\hline 2 & 92 & 100 & 0 & 0 & 0 & 0 & 8 & 0 \\
\hline 3 & 43 & 85 & 23 & 0 & 19 & 0 & 15 & 15 \\
\hline 4 & 88 & 100 & 0 & 0 & 8 & 0 & 4 & 0 \\
\hline 5 & 62 & 85 & 0 & 0 & 31 & 15 & 8 & 0 \\
\hline 6 & 34 & 54 & 50 & 46 & 8 & 0 & 8 & 0 \\
\hline 7 & 84 & 85 & 0 & 0 & 4 & 15 & 12 & 0 \\
\hline
\end{tabular}

Como se puede observar en la tabla 1, los estudiantes de género femenino tienen una mayor capacidad de relacionar situaciones, ya sean gráficas o literales, con los conceptos que se han desarrollado al interior del aula de clases, lo que permite inferir que las estudiantes pueden abstraer y relacionar más fácilmente, a diferencia de los estudiantes hombres. También se observa que las estudiantes siempre encontraron una relación que fuese evidente con el concepto analizado, en contraposición con los estudiantes hombres, quienes hicieron interpretaciones erróneas del modelo propuesto y malas interpretaciones del mismo; también hubo estudiantes hombres que no lograron interpretar el modelo o analogía propuesta. 
Tabla 2. Concepción acerca de lo que es una analogía.

\begin{tabular}{|c|c|c|c|c|}
\hline \multirow{2}{*}{$\begin{array}{c}\text { Pregunta 8 } \\
\text { Hombre }\end{array}$} & $58 \%$ & $3 \%$ & $27 \%$ & Opción C \\
\hline Mujer & $77 \%$ & $0 \%$ & $0 \%$ & $23 \%$ \\
\hline Pregunta 9 & Opción A & Opción B & Opción C & Opción D \\
\hline Hombre & $19 \%$ & $38 \%$ & $35 \%$ & $8 \%$ \\
\hline Mujer & $0 \%$ & $23 \%$ & $77 \%$ & $0 \%$ \\
\hline Pregunta 10 & Opción A & Opción B & Opción C & Opción D \\
\hline Hombre & $58 \%$ & $38 \%$ & $0 \%$ & $4 \%$ \\
\hline Mujer & $54 \%$ & $46 \%$ & $0 \%$ & $0 \%$ \\
\hline
\end{tabular}

El análisis de la tabla 2 muestra diferencias importantes en cuanto a las concepciones de los estudiantes hombres y mujeres, en torno a lo que son las analogías. En general las estudiantes mujeres tienen un pensamiento más crítico y racional, ya que identifican claramente qué es una analogía y qué presentaciones puede adoptar esta.

Tabla 3. Prospectiva del uso de analogías en la enseñanza de la Química.

\begin{tabular}{|c|c|c|c|}
\hline \multirow{2}{*}{$\begin{array}{c}\text { Pregunta 11 } \\
\text { Hombre }\end{array}$} & $85 \%$ & $15 \%$ & No lo sé \\
\hline Mujer & $85 \%$ & $15 \%$ & $0 \%$ \\
\hline Pregunta 12 & Sí & No & No lo sé \\
\hline Hombre & $88 \%$ & $0 \%$ & $12 \%$ \\
\hline Mujer & $92 \%$ & $0 \%$ & $8 \%$ \\
\hline Pregunta 13 & Si & No & No lo sé \\
\hline Hombre & $46 \%$ & $46 \%$ & $28 \%$ \\
\hline Mujer & $69 \%$ & $31 \%$ & $0 \%$ \\
\hline
\end{tabular}

El análisis de la tabla 3 muestra que a la gran mayoría de estudiantes se les facilita el aprendizaje de la Química cuando se ponen a consideración varias analogías que tengan algún tipo de relación con la temática analizada. 


\section{Conclusiones}

El uso de analogías y modelos analógicos en la enseñanza de la Química evidenció un cambio en el nivel de abstracción y en la forma de relacionar los conceptos, principios y teorías por parte de los estudiantes.

Las analogías o modelos analógicos son un excelente medio para construir y reconstruir conceptos químicos, ya que facilita el análisis de la situación o fenómeno real.

El concepto de analogía, en el contexto de la enseñanza de las ciencias, es más amplio y complejo que el que manejan las personas del común, puesto que para ellos las analogías son simples juegos de palabras que en el fondo tienen alguna relación, mientras que para una comunidad de especialistas, en el área de la investigación de los procesos de enseñanza-aprendizaje, ésta es concebida como una forma de expresión que permite representaciones más significativas del contenido de una temática y ayuda a la transferencia de este a otros campos.

\section{Bibliografía}

Arcá, M. y Guidoni, P. (1989). Modelos infantiles y modelos científicos sobre la morfología de los seres vivos. Enseñanza de las ciencias, 7(2), 162-167.

Campanario, J.M. y Moya, A. (1999). ¿Cómo enseñar ciencias? Principales tendencias y propuestas. Enseñanza de las ciencias, 17 (2), 179-192.

Galagovsky, L. y Adúriz-Bravo, A. (2001). Modelos y analogías en la enseñanza de las ciencias naturales. El concepto de modelo didáctico analógico. Enseñanza de las ciencias, 19(2), 231-242.

Gallego, R. y Pérez, R. (2003). El problema del cambio en las concepciones epistemológicas, pedagógicas y didácticas. Bogotá: Universidad Pedagógica Nacional, ARFO editores e impresores.

Gutiérrez, R. (1987). Psicología y aprendizaje de las ciencias. El modelo de Ausubel. Enseñanza de las ciencias, 5(2).

Oliva, J.M. (1999). Algunas reflexiones sobre las concepciones alternativas y el cambio conceptual. Enseñanza de las ciencias, (1), 93-107.

Oliva, J.M., Aragón, M.M., Mateo, J. y Bonat, M. (2001). Una propuesta didáctica basada en la investigación para el uso de analogías en la enseñanza de las ciencias. Enseñanza de las ciencias, 19 (3), 453-470. 


\section{UNIVERSIDAD PEDAGÓGICA NACIONAL PROYECTO DE PRÁCTICA PEDAGÓGICA Y DIDÁCTICA II}

La siguiente prueba no tiene carácter valorativo. Respóndala con la mayor sinceridad posible

1. Cuando un gas, por ejemplo un perfume, el olor a galletas recién horneadas o el vapor de un reactivo químico se difunde en una habitación se debe a:
a) Corrientes de aire que están presentes en la habitación
b) La energía cinética que posee una molécula
c) A la estructura atómica o molecular de dicho gas
d) Ninguna de las anteriores

2. Los gases que no se pueden percibir por medio del olfato, se deben a que:
a) No existen
b) No están compuestos por átomos o moléculas
c) Su estructura atómica les confiere dicha propiedad
d) Ninguna de las anteriores

3. El movimiento de una molécula de un gas en un recipiente se asemeja a:
a) El movimiento de una mariposa en pleno vuelo
b) El movimiento de la luna alrededor del sol
c) El recorrido de un balón de fútbol disputado en un partido
d) Ninguna de las anteriores

4. Una visión molecular de los gases sería similar a:
a) Una cantidad de canicas dispuestas ordenadamente en una caja
b) Gotas de lluvia en pleno aguacero
c) El vuelo caótico de una bandada de murciélagos
d) Ninguna de las anteriores

5. La Ley de Boyle (relación entre presión y volumen), la relaciono con:
a) El funcionamiento de una jeringa
b) El proceso de inflado de un balón
c) El fenómeno que ocurre con el ascenso de un globo meteorológico
d) Ninguna de las anteriores 
6. Cuando se habla del proceso de ebullición, me hago una imagen mental de:
a) Moléculas de una sustancia saliendo de su fase líquida
b) Un líquido calentándose en una estufa
c) Una sustancia que pasa de estado líquido a estado sólido en un congelador
d) Ninguna de las anteriores

7. Para usted, una analogía es:
a) Una relación entre dos situaciones similares
b) Una representación gráfica de un concepto
c) Una comparación entre un hecho conocido y uno desconocido
d) Todos los anteriores

8. Muchos conceptos químicos son complejos y abstractos. Por ejemplo el concepto de átomo. ¿Cómo cree que se debería enseñar esta temática?
a) Leyendo solo la teoría
b) Mediante videos
c) Mediante modelos y analogías
d) No lo sé

9. ¿Cuál de las siguientes alternativas escogería para analizar una temática específica?
a) Análisis de una situación real
b) Análisis mediante modelos gráficos
c) Análisis de lecturas
d) No lo sé

10. ¿Cree que con el uso de analogías se facilita el aprendizaje de la Química?

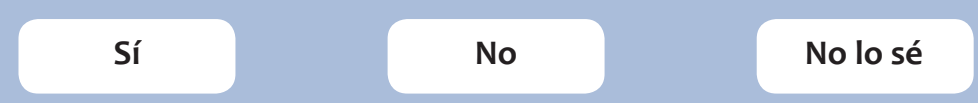

11. El concepto de mezclas se asimila mejor cuando visualizamos una imagen o una representación real de dicho concepto.
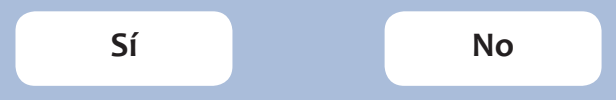

No lo sé

12. ¿La enseñanza de la Química debería partir de situaciones cotidianas?

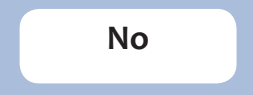

No lo sé 